\title{
BANACH SPACES AND ALGEBRAS OF CONTINUOUS FUNCTIONS ${ }^{1}$
}

\author{
ROBERT MACDOWELL
}

1. Introduction. If $X$ is a Hausdorff space, then we shall denote by $C(X)$ the set of real, bounded, continuous functions defined on $X$. If we define addition and scalar multiplication of functions in the usual way, then $C(X)$ becomes a group with real operator domain. The function which is identically zero on $X$ is the additive identity of this group, and we denote it by $\theta$. Under the norm $\|f\|=\sup _{x}|f(x)|$ this space becomes a real Banach space. Finally, provided with the usual multiplication of functions, this space becomes a real Banach algebra. The function which is identically one on $X$ is the multiplicative identity, and we denote it by $e$.

According to the Banach-Stone theorem [5], ${ }^{2}$ if $X$ is compact, then the topology of $X$ is reflected in the algebraic and metric structure of $C(X)$ in the sense that if $C(X)$ and $C(Y)$ are equivalent as Banach spaces, then $X$ is homeomorphic to $Y$. This general structural relation suggests particularization: what are the specific algebraic or metric properties of $C(X)$ which correspond to specific topological features of $X$ ? Significant results have been obtained in this direction by Myers, Eilenberg, and others $[1 ; 2 ; 3 ; 4]$.

This paper is drawn along these lines, and is concerned in particular with those spaces of continuous functions which are defined on the topological product of two compact Hausdorff spaces, or on compact Hausdorff spaces which contain such a product. ${ }^{8}$ The central problems are those of characterization; for the cases of products, and spaces which contain a product subspace, characterizations have been obtained. I have also obtained characterizations of continuous functions spaces over fiber spaces and fiber bundles, but the results are quite technical and not very revealing, and therefore are not of sufficient interest to include here.

Received by the editors March 10, 1954.

1 This paper is a part of the author's dissertation submitted in partial fulfillment of the requirements of the degree of Doctor of Philosophy at the University of Michigan. The author is indebted to Professor S. B. Myers who directed the progress of this dissertation, and to the Office of Naval Research under whose contract No. N8-ONR71400 part of this dissertation was completed.

2 Numbers in brackets refer to the bibliography.

' In this paper the only continuous function spaces we shall consider are those defined over compact Hausdorff spaces. 


\section{Subspaces of $C(X)$.}

Definition. A set of elements $T$ drawn from a Banach space $B$ maximal with respect to the property $b_{1}, \cdots, b_{n} \in T \rightarrow\left\|\sum_{i=1}^{n} b_{i}\right\|$ $=\sum_{i=1}^{n}\left\|b_{i}\right\|$ is called a $T$-set of $B$.

The $T$-sets of a Banach space were introduced by Myers [2], and their significant features were enunciated by him. We shall use that $T$-sets exist, cover the space $B$, are positively homogeneous, and are convex. Also, if the Banach space $B$ is a space of continuous functions, say $B=C(X)$, where $X$ is compact, then Myers has shown that every $T$-set in $B$ has the form

$$
\left\{f \in B \mid f\left(x_{0}\right)=\|f\|\right\} \quad \text { or } \quad\left\{f \in B \mid f\left(x_{0}\right)=-\|f\|\right\},
$$

and conversely, every such subset is a $T$-set of $B$. If we denote the first of these $T$-sets by $T\left(x_{0}\right)$, then it is easily seen that the second has the form $-T\left(x_{0}\right)$.

Definition. A subspace $F$ of a Banach space $B$ is said to be $T$ normally imbedded in $B$ if for every $T$-set $T$ of $B, T \cap F$ is a $T$-set of F.

We shall be concerned with the $T$-normal subspaces of a Banach space, and criteria for recognizing them are therefore of interest:

T1. A subspace $F$ is $T$-normally imbedded in $B$ if and only if every $T$-set of $B$ contains a $T$-set of $F$.

T2. If $F$ is a subspace of $C(X)$ which contains the constant functions, and is closed under absolute value, then $F$ is $T$-normal in $C(X)$.

The first of these conditions follows almost immediately from the maximal character of $T$-sets, and to prove the second we proceed as follows:

Suppose that $F$ is not $T$-normal in $C(X)$. Then there exists a $T$-set $T$ of $C(X)$ such that $T \cap F$ is not a $T$-set of $F$. Thus, there exists $f \in F$ such that $\|f+g\|=\|f\|+\|g\|$ for every $g \in T \cap F$, but $f \notin T$. Let us suppose that $T=T\left(x_{0}\right)$ (the argument is similar if $T=-T\left(x_{0}\right)$ ).

Now, $\left|f\left(x_{0}\right)\right|<\|f\|$, for if $f\left(x_{0}\right)=\|f\|$ then $f \in T$, contrary to assumption, and if $-f\left(x_{0}\right)=\|f\|$, then $-f \in T$ so that $\|f+(-f)\|$ $=2\|f\|=0$, whence $f=\theta$, a contradiction since $\theta$ is contained in every $T$-set. We can suppose that $f\left(x_{0}\right) \geqq 0$, for the argument is strictly analogous if $f\left(x_{0}\right) \leqq 0$.

Let $h(x)=f(x)-f\left(x_{0}\right), m(x)=|h(x)|$, and $n(x)=\|m\|-m(x)$. Since $F$ is a subspace which contains the constant functions and is closed under absolute value, the functions $h, m$, and $n$ are contained in $F$, and $n \neq \theta$. 
Now one easily checks that $n\left(x_{0}\right)=\|n\|$ so that $n \in T \cap F$, whence by assumption $\|f+n\|=\|f\|+\|n\|$. However this relation implies that both $f$ and $n$ are in some $T$-set, and since $n(x) \geqq 0$, it must have the form $T(\bar{x})$. Hence $f(\bar{x})=\|f\|$, and $n(\bar{x})=\|n\|$. But we then have $f(\bar{x})>f\left(x_{0}\right)$ so that both $h(\bar{x})>0$ and $m(\bar{x})>0$. This however yields $n(\bar{x})<\|n\|$, a contradiction, and the theorem is therefore proved.

Definition. If $T$ is a $T$-set of a Banach space $B$, then the set $S=T \cup-T$ is called a symmetricized $T$-set of $B$, denoted by an $s T$-set.

One easily verifies that a $T$-normal subspace is $s T$-normal. More precisely:

T3. If $F$ is a $T$-normal subspace of $B$, and $S$ is an $s T$-set of $B$, then $S \cap F$ is an $s T$-set of $F$.

Our concern now will not be so much with the orientation of a single subspace in a Banach space, but rather with the mutual orientation of several subspaces with each other. In particular three possible mutual orientations will be interesting:

Definition. If $F$ and $G$ are closed subspaces of a Banach space $B$ with the property that $f \in F, g \in G$ imply either $\|f+g\|=\|f\|+\|g\|$ or $\|f-g\|=\|f\|+\|g\|$, then we say that $F$ and $G$ are additively related.

Definition. If $F$ and $G$ are closed subspaces of a Banach space $B$ with the property that for any pair of $s T$-sets $S_{F}, S_{G}$ of $F$ and $G$ respectively there exists an $s T$-set $S$ of $B$ such that $S_{F} \cup S_{G} \subset S$, then we say that $F$ and $G$ are $S$-admittant in $B$.

Definition. If $F$ and $G$ are closed subspaces of a Banach space $B$ with the property that for any pair of $s T$-sets $S_{F}, S_{G}$ of $F$ and $G$ respectively there exists not more than one $s T$-set $S$ of $B$ such that $S_{F} \cup S_{G} \subset S$, then we say that $F$ and $G$ are $S$-determinant in $B$.

These definitions are not unrelated, in fact

T4. If $F$ and $G$ are additively related subspaces of $C(X)$, then they are $S$-admittant in $C(X)$.

Proof. Let us suppose that $F$ and $G$ are additively related but are not $S$-admittant, Then there exist $s T$-sets $S_{F}$ and $S_{G}$ of $F$ and $G$ respectively such that $S_{F} \cup S_{G}$ is contained in no $s T$-set of $C(X)$.

Let $S_{F}=T_{F} \cup-T_{F}$ and $S_{G}=T_{G} \cup-T_{G}$. Note that neither $T_{F} \cup T_{G}$ nor $T_{F} \cup-T_{G}$ is contained in a $T$-set of $C(X)$ for otherwise we should have $S_{F} \cup S_{G}$ contained in an $s T$-set of $C(X)$. Hence, there exist $f_{F}, \bar{f}_{F} \in T_{F}$ and $f_{G}, \bar{f}_{G} \in T_{G}$ such that $\left\|f_{F}+f_{G}\right\|<\left\|f_{F}\right\|+\left\|f_{G}\right\|$ and $\left\|\bar{f}_{F}-\bar{f}_{G}\right\|<\left\|\bar{f}_{F}\right\|+\left\|\bar{f}_{G}\right\|$. If we let $g_{F}=f_{F}+\bar{f}_{F}$ and $h_{G}=f_{G}+\bar{f}_{G}$, a simple computation shows that $\left\|g_{F}+h_{G}\right\|<\left\|g_{F}\right\|+\left\|h_{G}\right\|$ and $\left\|g_{F}-h_{G}\right\|$ $<\left\|g_{F}\right\|+\left\|h_{G}\right\|$. Since however $g_{F} \in F$ and $h_{G} \in G$ we see that $F$ and $G$ 
are not additively related, contrary to assumption. Hence the theorem is proved.

Now we turn to an investigation of these concepts in terms of the algebraic structure of $C(X)$.

\section{Subalgebras of $C(X)$.}

Definition. A subalgebra of $C(X)$ which contains the unit $e$ is called an analytic subalgebra of $C(X)$.

T5. If $F$ is an analytic subalgebra of $C(X)$, then $F$ is $T$-normal in $C(X)$.

Proof. We assume that $F$ is not $T$-normal, and then as in T2 we establish the existence of a function $f$ such that $\|f+g\|=\|f\|+\|g\|$ for every $g \in T \cap F$, but $f \notin T$. If we let $T=T\left(x_{0}\right)$, then we may assert that $\left|f\left(x_{0}\right)\right|<\|f\|$.

We now let $h(x)=f(x)-f\left(x_{0}\right), m(x)=(h(x))^{2}$, and $n(x)=\|m\|-m(x)$. Proceeding exactly as in T2 we show that there exists $\bar{x} \in X$ such that $f(\bar{x})=\|f\|$ and $n(\bar{x})=\|n\|$. By the construction of $n$ however we have $n(\bar{x})<\|n\|$, and this contradiction establishes the theorem.

We now turn to characterizing $S$-determinancy within the algebraic and metric structure of $C(X)$.

Definition. A subset $N$ of the space $C(X)$ is said to separate $X$ if for every $x$ and $\bar{x}$ in $X$ there exists a function $f$ in $N$ such that $f(x)$ $\neq f(\bar{x})$.

T6. If $A_{1}$ and $A_{2}$ are analytic subalgebras of $C(X)$, then $A_{1}$ and $A_{2}$ are $S$-determinant in $C(X)$ if and only if $A_{1} \cup A_{2}$ separates $X$.

Proof. (a) Suppose that $A_{1} \cup A_{2}$ separates $X$. Let $S_{1}$ and $S_{2}$ be $s T$-sets of $A_{1}$ and $A_{2}$ respectively, and suppose that $S_{1} \cup S_{2} \subset S(\bar{x})$, and $S_{1} \cup S_{2} \subset S\left(x^{*}\right)$, where $x \neq x^{*}$. Since $A_{1} \cup A_{2}$ separates $X$, there exists $g \in A_{1} \cup A_{2}$, say $g \in A_{1}$, such that $g(\bar{x}) \neq g\left(x^{*}\right)$. Let $h(x)=-[g(x)$ $-g(\bar{x})]^{2}$, and let $f(x)=h(x)+\|h\|$. Since $A_{1}$ is analytic, $f$ is contained in $A_{1}$, and by virtue of the definition of $f, f(\bar{x})=\|f\|, f\left(x^{*}\right)<\|f\|$, and $f(x) \geqq 0$. Therefore $f \in S(\bar{x}) \cap A_{1}=S_{1}$, and $f \in S\left(x^{*}\right)$. Hence $S_{1} \cup S_{2}$ $\llbracket S\left(x^{*}\right)$, a contradiction. We have then that $S_{1} \cup S_{2}$ is contained in not more than one $s T$-set of $C(X)$.

(b) Suppose that for any pair of $s T$-sets $S_{1}$ and $S_{2}$ of $A_{1}$ and $A_{2}$ respectively there exists not more than one $s T$-set $S$ of $C(X)$ such that $S_{1} \cup S_{2} \subset S$. Let $\bar{x}, x^{*} \in X, \bar{x} \neq x^{*}$, and let $\bar{S}_{i}=S(\bar{x}) \cap A_{i}$ and $S_{i}^{*}=S\left(x^{*}\right) \cap A_{i}, \quad i=1,2$. Now, by assumption either $\bar{S}_{1} \neq S_{1}^{*}$ or $\bar{S}_{2} \neq S_{2}^{*}$, say $\bar{S}_{1} \neq S_{1}^{*}$. Hence, there exists $f \in \bar{S}_{1}, f \in S_{1}^{*}$ or there exists $f \in S_{1}^{*}, f \notin \bar{S}_{1}$. Suppose the first case obtains. But $f \in \bar{S}_{1}$ implies that 
$|f(\bar{x})|=\|f\|$, and $f \notin S_{1}^{*}$ implies that $\left|f\left(x^{*}\right)\right|<\|f\|$, so that $f$ separates $\bar{x}$ and $x^{*}$.

Definition. If $N$ is a subset of the algebra $C(X)$, let $(N)_{A}$ denote the smallest subalgebra of $C(X)$ which contains $N$.

T7. A subset $F$ of $C(X)$ which contains e separates $X$ if and only if $(F)_{A}$ is dense in $C(X)$.

Proof. (a) If $F$ separates $X$, by the Weierstrass-Stone theorem [5], $(F)_{A}$ is dense in $C(X)$.

(b) If $F$ does not separate $X$, there exist $x, \bar{x}$ contained in $X$, $x \neq \bar{x}$, such that $f(x)=f(\bar{x})$ for every $f$ contained in $F$. Hence $q(x)$ $=q(\bar{x})$ for every $q \in(F)_{A}$. Since however $C(X)$ is completely regular it follows that $(F)_{A}$ is not dense in $C(X)$.

Now we may summarize the results of the last several theorems in a rather nice characterization of $S$-determinancy within $C(X)$ :

T8. If $A_{1}$ and $A_{2}$ are analytic subalgebras of $C(X)$, then $A_{1}$ and $A_{2}$ are $S$-determinant in $C(X)$ if and only if the closure of the algebra generated by $A_{1}$ and $A_{2}$ is $C(X)$.

The proof of this theorem follows from the results of T6 and T7.

4. The algebra $C(X)$ where $X$ is a topological product. In this section we shall let $X_{1}$ and $X_{2}$ be compact Hausdorff spaces, and shall let $X$ denote their topological product. In accord with the previous notation the spaces (algebras) of real continuous functions defined on these spaces will be denoted by $C(X)$ and $C\left(X_{i}\right), i=1,2$.

Definition. $g \in C(X)$ will be called a semi-constant function of type (1) if

$$
\left(x_{1}, x_{2}\right),\left(x_{1}, \bar{x}_{2}\right) \in X \rightarrow g\left(x_{1}, x_{2}\right)=g\left(x_{1}, \bar{x}_{2}\right),
$$

and of type (2) if

$$
\left(x_{1}, x_{2}\right),\left(\bar{x}_{1}, x_{2}\right) \in X \rightarrow g\left(x_{1}, x_{2}\right)=g\left(\bar{x}_{1}, x_{2}\right) .
$$

Let $F_{i}$ denote the collection of semi-constant functions of type $(i)$. One may easily verify that the collections $F_{i}$ are analytic subalgebras of $C(X)$, and then from the observation that each $F_{i}$ is equivalent as a Banach algebra to $C\left(X_{i}\right)$ we may conclude that the $F_{i}$ are complete, and therefore closed in $C(X)$. For purposes of reference then:

T9. The collections $F_{i}$ of semi-constant functions of type (i) are closed subalgebras of $C(X), i=1,2$. 
Definition. Let $f \in C(X)$. Then the norm set of $f, N(f)=\{x \in X \mid$ $|f(x)|=\|f\|\}$.

T10. $F_{1}$ and $F_{2}$ are additively related.

Proof. Let $f_{i} \in F_{i}, i=1,2$. Since $X$ is compact, there exist $x, y \in X$ such that $\left|f_{1}(x)\right|=\left\|f_{1}\right\|$ and $\left|f_{2}(y)\right|=\left\|f_{2}\right\|$. If now $x=\left(x_{1}, x_{2}\right)$ and $y=\left(y_{1}, y_{2}\right)$ we have from the definition of the $F_{i}$ that $\left|f_{1}\left(x_{1}, y_{2}\right)\right|$ $=\left|f_{1}\left(x_{1}, x_{2}\right)\right|=\left\|f_{1}\right\|$, and $\left|f_{2}\left(y_{1}, y_{2}\right)\right|=\left|f_{2}\left(x_{1}, y_{2}\right)\right|=\left\|f_{2}\right\|$, or that $\bar{x}$ $=\left(x_{1}, y_{2}\right) \in N\left(f_{1}\right) \cap N\left(f_{2}\right)$. Now there are essentially two cases: where both $f_{1}(\bar{x})$ and $f_{2}(\bar{x})$ are non-negative, and where one of $f_{1}(\bar{x}), f_{2}(\bar{x})$ is negative.

(a) If $f_{1}(\bar{x}) \geqq 0$ and $f_{2}(\bar{x}) \geqq 0$, then $f_{1}(\bar{x})=\left\|f_{1}\right\|$ and $f_{2}(\bar{x})=\left\|f_{2}\right\|$ so that $f_{1}, f_{2} \in T(\bar{x})$, whence $\left\|f_{1}+f_{2}\right\|=\left\|f_{1}\right\|+\left\|f_{2}\right\|$.

(b) If $f_{1}(\bar{x}) \geqq 0$ and $f_{2}(\bar{x}) \leqq 0$, then $f_{1}(\bar{x})=\left\|f_{1}\right\|$ and $-f_{2}(\bar{x})=\left\|f_{2}\right\|$ so that $f_{1}, \quad-f_{2} \in T(\bar{x})$, whence $\left\|f_{1}-f_{2}\right\|=\left\|f_{1}+\left(-f_{2}\right)\right\|=\left\|f_{1}\right\|+\left\|-f_{2}\right\|$ $=\left\|f_{1}\right\|+\left\|f_{2}\right\|$.

Thus, for every pair of functions $f_{1}$ and $f_{2}$ contained respectively in $F_{1}$ and $F_{2}$ we have either $\left\|f_{1}+f_{2}\right\|=\left\|f_{1}\right\|+\left\|f_{2}\right\|$ or $\left\|f_{1}-f_{2}\right\|=\left\|f_{1}\right\|+\left\|f_{2}\right\|$, so that the algebras $F_{1}$ and $F_{2}$ are additively related.

T11. $F_{1}$ and $F_{2}$ are $S$-determinant in $C(X)$.

Proof. By virtue of T6 and T9 we need only show that $F_{1} \cup F_{2}$ separates $X$. Let $x, \bar{x} \in X, x \neq \bar{x}$, where $x=\left(x_{1}, x_{2}\right), \bar{x}=\left(\bar{x}_{1}, \bar{x}_{2}\right)$. Since either $x_{1} \neq \bar{x}_{1}$, or $x_{2} \neq \bar{x}_{2}$, let us suppose that $x_{1} \neq \bar{x}_{1}$. Because $C\left(X_{1}\right)$ is completely regular, there exists $\bar{f} \in C\left(X_{1}\right)$ such that $\bar{f}\left(x_{1}\right) \neq \bar{f}\left(\bar{x}_{1}\right)$. We define $f\left(x_{1}, x_{2}\right)=\bar{f}\left(x_{1}\right)$, and note that $f \in F_{1}$. Since, however, $f\left(x_{1}, x_{2}\right)$ $=\bar{f}\left(x_{1}\right) \neq \bar{f}\left(\bar{x}_{1}\right)=f\left(\bar{x}_{1}, \bar{x}_{2}\right), f$ separates $x$ and $\bar{x}$ and the theorem is therefore proved.

Thus, to summarize briefly: the collections $F_{i}$ of semi-constant functions of type $(i)$ on $X$ are additively related, $S$-determinant closed analytic subalgebras of $C(X)$.

5. Characterization of the space (algebra) $C\left(X_{1} \times X_{2}\right)$. In this section we shall let $S$ denote the class of $s T$-sets of $C(X)$, and $S_{i}$ the class of $s T$-sets of a closed subspace $F_{i}$ of $C(X)$. For $f \in C(X)$ and $f_{i} \in F_{i}$ let $C(f)=\{S \in \mathcal{S} \mid f \in S\}$, and $C_{i}\left(f_{i}\right)=\left\{S_{i} \in \mathcal{S}_{i} \mid f_{i} \in S_{i}\right\}$. Let $\Lambda=\{C(f) \mid f \in C(X)\}$, and $\Lambda_{i}=\left\{C_{i}\left(f_{i}\right) \mid f_{i} \in F_{i}\right\}$.

The collections of subsets $\Lambda$ and $\Lambda_{i}$ of $S$ and $S_{i}$ respectively may be considered as generators for systems of closed subsets of $S$ and $S_{i}$ respectively, thus providing these sets with topologies. We shall call these the normal topologies, and shall assume hereafter that $S$ and $S_{i}$ carry the normal topologies. 
T12. The space $S$ is homeomorphic to $X$.

Proof. Except for changes in notation this result is contained in Eilenberg's paper [1].

T13. Let $C(X)$ be the Banach space of real continuous functions defined on the compact space $X$. Then $X$ is a product space if and only if $C(X)$ contains two $T$-normal, additively related closed subspaces which are $S$-determinant in $C(X)$.

Proof. The necessity of these conditions has been established in T9, T5, T10, and T11.

By T12 the sufficiency of the conditions will be demonstrated if we can show that $S$ is a product space. We shall do this by exhibiting a homeomorphism of $S$ onto $S_{1} \times S_{2}$, where $S_{1}$ and $S_{2}$ are the classes of $s T$-sets of the subspaces $F_{1}$ and $F_{2}$ whose existence is asserted in the hypotheses of the theorem.

We define the mapping $F: S \rightarrow S_{1} \times \mathcal{S}_{2}$, where $F(S)=\left(S \cap F_{1}\right.$, $\left.S \cap F_{2}\right)$. By the $T$-normality of the $F_{i}$, this mapping is well defined (see T3).

(a) $F$ is onto all of $S_{1} \times S_{2}:$ By T4, $F_{1}$ and $F_{2}$ are $S$-admittant in $C(X)$. Hence $S_{i} \in \mathcal{S}_{i}, i=1$ and 2 , implies there exists $S \in \mathcal{S}$ such that $S_{1} \cup S_{2} \subset S$. Now, I claim that $S_{1}=S \cap F_{1}$ and $S_{2}=S \cap F_{2}$, for suppose that $S_{1}=T_{1} \cup-T_{1}$ and $S=T \cup-T$. Then $T_{1} \cup-T_{1} \subset T \cup-T$ from which we may conclude that either $T_{1} \subset T$ or $T_{1} \subset-T$ for, if not, there exists $f \in T_{1}, f \notin T$, and there exists $g \in T_{1}, g \notin-T$. Since $T_{1}$ is positively homogeneous $f+g \in T_{1}$, and hence $f+g$ must be contained in either $T$ or $-T$. Suppose that $f+g \in T$. Since $-f \in T$ we have $\|(f+g)+(-f)\|=\|f+g\|+\|-f\|=2\|f\|+\|g\|=\|g\|$, so that $f=\theta$, a contradiction since $\theta \in T$. On the other hand suppose that $f+g \in-T$. Since $-g \in-T$ we have similarly that $\|(f+g)+(-g)\|=\|f+g\|$ $+\|-g\|=\|f\|+2\|g\|=\|f\|$, so that $g=\theta$, a contradiction since $\theta \in-T$. We have then that either $T_{1} \subset T$ or $T_{1} \subset-T$. Let us suppose that $T_{1} \subset T$ whence $T_{1} \subset T \cap F_{1}$. Now, by the additivity of $T \cap F_{1}$, and the maximality of $T_{1}, T_{1}=T \cap F_{1}$. Hence, $T_{1} \cup-T_{1}=\left(T \cap F_{1}\right) \cup\left(-T \cap F_{1}\right)$ $=(T \cup-T) \cap F_{1}=S \cap F_{1}$, so that $S_{1}=S \cap F_{1}$. Analogously we have that $S_{2}=S \cap F_{2}$. Hence, $F(S)=\left(S_{1}, S_{2}\right)$, and $F$ is therefore onto.

(b) $F$ is 1-1: If $S \cap F_{i}=\bar{S} \cap F_{i}$ for $i=1$ and 2, then $\left(S \cap F_{1}\right)$ $\cup\left(S \cap F_{2}\right) \subset S$ and $\left(S \cap F_{1}\right) \cup\left(S \cap F_{2}\right) \subset \bar{S}$. But, by the $T$-normality of the $F_{i}, S \cap F_{i} \in S_{i}$ for $i=1$ and 2 , and hence by the $S$-determinancy of the $F_{i}, S=\bar{S}$. Hence $F(S)=F(\bar{S})$ implies that $S=\bar{S}$, and $F$ is therefore 1-1.

Now, since $S$ is compact, in order to show that $F$ is a homeomor- 
phism it is sufficient to show that it is continuous.

(c) $F$ is continuous: We shall show that the inverse image of any set in the closed sub-base of $S_{1} \times S_{2}$ is a member of the closed base of $S$. In particular, for $f_{i} \in F_{i}, i=1$ and 2 , we shall show that there exists $f \in C(X)$ such that $C(f)=F^{-1}\left(C_{1}\left(f_{1}\right), C_{2}\left(f_{2}\right)\right)$.

Since the $F_{i}$ are additively related, either

$$
\left\|f_{1}+f_{2}\right\|=\left\|f_{1}\right\|+\left\|f_{2}\right\|, \text { or }\left\|f_{1}-f_{2}\right\|=\left\|f_{1}\right\|+\left\|f_{2}\right\| .
$$

(1) Suppose that $\left\|f_{1}+f_{2}\right\|=\left\|f_{1}\right\|+\left\|f_{2}\right\|$. In this case we let $f=f_{1}+f_{2}$, and must show then that $C(f)=F^{-1}\left(C_{1}\left(f_{1}\right), C_{2}\left(f_{2}\right)\right)$.

(i) $F^{-1}\left(C_{1}\left(f_{1}\right), \quad C_{2}\left(f_{2}\right)\right) \subset C(f)$ : Let $S \in F^{-1}\left(C_{1}\left(f_{1}\right), C_{2}\left(f_{2}\right)\right)$. Then $f_{i} \in S \cap F_{i}$, and hence $f_{1}, f_{2} \in S$. Since $\left\|f_{1}+f_{2}\right\|=\left\|f_{1}\right\|+\left\|f_{2}\right\|, f_{1}$ and $f_{2}$ are in the same $T$-set of $S$. Hence $f_{1}+f_{2}$ is in this $T$-set, and therefore in $S$. But $f \in S$ means that $S \in C(f)$.

(ii) $C(f) \subset F^{-1}\left(C_{1}\left(f_{1}\right), C_{2}\left(f_{2}\right)\right)$. Let $S \in C(f)$. Hence $f=f_{1}+f_{2} \in S$. Now, since $F(S)=\left(S \cap F_{1}, S \cap F_{2}\right)$, if we can show that $f_{1}$ and $f_{2}$ are in $S$ we are through, for $f_{i} \in F_{i}, f_{i} \in S$ implies that $f_{i} \in S \cap F_{i}$ which implies that $S \cap F_{i}$ are contained in $C_{i}\left(f_{i}\right)$ which in turn implies that $S \in F^{-1}\left(C_{1}\left(f_{1}\right), C_{2}\left(f_{2}\right)\right)$.

We shall show that $f_{1} \in S$, and note that the argument is similar for $f_{2}$.

Let $S=T \cup-T$, and suppose that $f \in T$. Then $f_{1}$ will be in $T$, and therefore in $S$, if $g \in T$ implies that $\left\|f_{1}+g\right\|=\left\|f_{1}\right\|+\|g\|$. This we shall show. Let $g \in T$. Now, $\left\|g+f_{1}\right\|=\left\|g+\left(f_{1}+f_{2}\right)-f_{2}\right\| \geqq\left\|g+\left(f_{1}+f_{2}\right)\right\|$ $-\left\|f_{2}\right\|=\|g\|+\left\|f_{1}\right\|$, so that $\left\|g+f_{1}\right\|=\|g\|+\left\|f_{1}\right\|$.

(2) Suppose that $\left\|f_{1}-f_{2}\right\|=\left\|f_{1}\right\|+\left\|f_{2}\right\|$. Rewriting this relation, $\left\|f_{1}+\left(-f_{2}\right)\right\|=\left\|f_{1}\right\|+\left\|-f_{2}\right\|$, whence by (1) we can assert that $C\left(f_{1}\right.$ $\left.+\left(-f_{2}\right)\right)=F^{-1}\left(C_{1}\left(f_{1}\right), C_{2}\left(-f_{2}\right)\right)$. But since $f_{i} \in S_{i}$ implies that $-f_{i} \in S_{i}$, $C_{2}\left(f_{2}\right)=C_{2}\left(-f_{2}\right)$, we have that $C\left(f_{1}-f_{2}\right)=F^{-1}\left(C_{1}\left(f_{1}\right), C_{2}\left(f_{2}\right)\right)$ which is the desired relation.

Thus, the proof is complete.

From T12 and the proof of T13 it is apparent that if the $F_{i}$ are equivalent as Banach spaces to $C\left(X_{i}\right)$, then $X$ is homeomorphic to $X_{1} \times X_{2}$. Of course, every space is trivially a product space, that is the product of itself with a single point, and this case arises when one of the $F_{i}$ is equivalent to the real numbers, and the other to $C(X)$. Thus, to eliminate this trivial case, we simply insist that neither of the $F_{i}$ be all of $C(X)$.

The characterization of Banach spaces of the form $C\left(X_{1} \times X_{2}\right)$ as expressed in T12, although satisfactory, involves somewhat artificial conditions: $T$-normality, and especially $S$-determinancy. When the 
algebraic structure of the space is brought into play, however, a far nicer characterization is possible.

T14. Let $C(X)$ be the Banach algebra of real, continuous functions defined on the compact space $X$. Then $X$ is a product space if and only if $C(X)$ contains two analytic subalgebras which are additively related, and the closure of whose generated subalgebra is $C(X)$.

Proof. The necessity of these conditions has been demonstrated in T9, T10, T11, and T8.

The sufficiency will be established if we can show that these conditions imply the hypotheses of T12. This follows however from T5 and T8.

The next problem we shall consider is that of characterizing the algebra of real continuous functions defined on a compact space $X$ which contains a closed subspace $Y$ in the form of a topological product. This problem however necessitates a brief investigation of the ideal structure in the algebra $C(X)$; we turn to this now.

6. Ideals in $C(X)$. Because frequent reference will be made to some basic properties of function algebras developed by Stone [5], we shall summarize some of his results in the form of a theorem, T15.

T15. Let $C(X)$ be an algebra of continuous functions on a compact space. Then corresponding to every closed ideal $I$ in $C(X)$ there exists a closed subset $K \subset X$ such that $I=\{f \in C(X) \mid f(K)=0\}$, and conversely for every closed subset $K \subset X$ the set $\{f \in C(X) \mid f(K)=0\}$ is a closed ideal in $C(X)$. The maximal ideals then have the form $I=I\left(x_{0}\right)$ $=\left\{f \in C(X) \mid f\left(x_{0}\right)=0\right\}$, and every closed ideal is the intersection of all maximal ideals in which it is contained. Moreover, if $I=I(K)$ is a closed ideal in $C(X)$, and we provide the quotient algebra $C(X) / I$ with the norm $\|b\|=\inf _{c \in b}\|c\|$, where $b$ is a coset in $C(X) / I$, then $C(X) / I$ is a Banach algebra equivalent to $C(K)$.

Now, let $J$ denote the class of maximal ideals in $C(X)$, and let $I$ be some closed ideal in $C(X)$. Let $\Im_{1}=\left\{I_{\alpha} \in J \mid I \subset I_{\alpha}\right\}$ and let $J_{2}=\left\{I_{\alpha} \in J \mid I_{\alpha} \notin J_{1}\right\}$. We define $I_{1}=\cap I_{\alpha} \mid I_{\alpha} \in J_{1}$ and $I_{2}=\cap I_{\alpha} \mid I_{\alpha} \in J_{2}$. By T14, we can assert that $I=I_{1}$. The closed ideal $I_{2}$ we shall call the ideal complementary to $I$, and denote it by $I_{C}$. Since $C(X)$ is without radical we might note that $C(X)$ and $(\theta)$ are mutually complementary ideals.

There are some elementary properties of these ideals which follow almost immediately from the above definitions.

T16. Let $I=I(x)$ be a maximal ideal and $I^{\prime}=(K)$ a closed ideal in 
$C(X)$, where $K$ is closed in $X$. Let $W$ be any subset of $X$. Then,

(1) $I^{\prime} \subset I$ if and only if $x \in K$,

(2) $I(W)=I(\mathcal{C}(W))$, where $I(W)=\{f \in C(X) \mid f(W)=0\}$, and $\mathcal{C}(W)$ denotes the closure of $W$,

(3) $I_{C}^{\prime}=I(C(C K))$, where $C K$ is the complement in $X$ of $K$.

Proof. (1) follows immediately from the normality of the space $X$. Since $W \subset \mathcal{C}(W)$ implies that $I(\mathcal{C}(W)) \subset I(W)$, to obtain (2) we need only show that $I(W) \subset I(C(W))$. This however follows from the continuity of the functions in $I(W)$. For (3) we may use the result (1): $I^{\prime} \nsubseteq I(x) \rightleftarrows x \notin K \rightleftarrows x \in C K$, so that $I_{C}^{\prime}=\cap I_{\alpha}\left|I_{\alpha} \in J_{1}=\bigcap I_{\alpha}\right| I^{\prime} \nsubseteq I_{\alpha}$ $=\bigcap I(x) \mid x \in C K=I(C K)$, whence by (2) $I_{C}^{\prime}=I(\bigodot(C K))$.

Now the importance of these observations is expressed in the

CoRollary. If $I=I(x)$ is a maximal ideal, and $I^{\prime}=I(K)$ is a closed ideal in $C(X)$, where $K$ is closed in $X$, then $x \in$ int $K$ if and only if $I_{C}^{\prime} \nsubseteq I$.

Proof. By (3) of T16, $I_{C}^{\prime}=I C(C K)$, and hence by (1) and the definition of the interior, $I_{C}^{\prime} \nsubseteq I(x) \rightleftarrows x \notin \mathcal{C}(C K) \rightleftarrows x \in C(\mathcal{C}(C K)) \rightleftarrows x \in$ int $K$.

For purposes of reference we express this relation somewhat differently:

T17. Let $I^{\prime}=I(K)$ be a closed ideal in $C(X)$ where $K$ is closed in $X$. Then int $K \neq \phi$ if and only if $I_{C}^{\prime} \neq(\theta)$.

Proof. int $K \neq \phi \rightleftarrows$ there exists $x \in$ int $K \rightleftarrows$ there exists $I(x)$ such that $I_{C}^{\prime} \nsubseteq I(x)$ by the corollary of T16. But equivalently then $I_{C}^{\prime} \neq(\theta)$, and the theorem is proved.

Note that T17 extends Stone's result as follows: the one-to-one correspondence between closed subsets of $X$ and the closed ideals of $C(X)$ as expressed in T15 establishes a one-to-one correspondence between those closed subsets of $X$ which have nonvacuous interiors and those ideals of $C(X)$ with non-null complements.

7. The algebra $C(X)$ where $X$ contains a topological product. In this section we shall be concerned with the characterization of the algebra of real continuous functions defined on a compact space $X$ which contains a closed subspace $Y$ homeomorphic to a topological product space. This problem is of course almost nonsense unless we have a method for determining the relative "size" of the subspace $Y$ in $X$. Thus, if $Y$ consists of four distinct points it is homeomorphic to a product space, and therefore $X$ contains a subset of the desired kind whenever it contains four or more points. We want to rule out this trivial case, and shall be insisting that $Y$ contain an interior in $X$. 
Let $Y$ be a closed subspace of $X$, and let $h$ be a homeomorphism of $X_{1} \times X_{2}$ onto $Y$, where $X_{1}$ and $X_{2}$ are compact spaces. Let $I=I(Y)$ be the closed ideal in $C(X)$ of all functions which vanish on $Y$. Then $C(X) / I$ is equivalent to $C(Y)$ as a Banach algebra, and hence by T13 contains two analytic subalgebras $F_{1}^{*}$ and $F_{2}^{*}$ which are additively related and the closure of whose generated subalgebra is $C(X) / I$. Let $H: C(X) \rightarrow C(X) / I$ be the natural homomorphism and let $F_{1}$ and $F_{2}$ be the analytic subalgebras $H^{-1}\left(F_{1}^{*}\right)$ and $H^{-1}\left(F_{2}^{*}\right)$ respectively.

T18. $C\left(\left(F_{1} \cup F_{2}\right)_{A}\right)=C(X)$.

Proof. Let $f \in C(X)$, and $e>0$. We shall show that there exists $g \in\left(F_{1} \cup F_{2}\right)_{A}$ such that $\|f-g\|<e$. Since $\mathcal{C}\left(\left(F_{1}^{*} \cup F_{2}^{*}\right)_{A}\right)=C(X) / I$, there exists $g^{*} \in\left(F_{1}^{*} \cup F_{2}^{*}\right)_{A}$ such that $\left\|g^{*}-H(f)\right\|<e$, i.e. $\sup _{Y} \mid g^{*}(y)$ $-f(y) \mid<e$. Let $h^{*}$ be the function defined on $Y$ as follows: $h^{*}(y)$ $=g^{*}(y)-f(y)$, and note that $\sup _{Y}\left|h^{*}(y)\right|<e$. Since $h^{*}$ is defined on a closed subset of the normal space $X$, there exists a norm preserving extension $h$ of $h^{*}$ over $X$. If we let $g(x)=h(x)+f(x)$, then $g(y)$ $=g^{*}(y)$ so that $g$ extends $g^{*}$, and

$$
\|f-g\|=\sup _{X}|f(x)-g(x)|=\sup _{X}|h(x)|<e .
$$

Since however $H(g)=g^{*} \in\left(F_{1}^{*} \cup F_{2}^{*}\right)_{A}, g \in H^{-1}\left(\left(F_{1}^{*} \cup F_{2}^{*}\right)_{A}\right)=\left(F_{1} \cup F_{2}\right)_{A}$, and the theorem is therefore proved.

Now it turns out that the existence of these algebras with the properties noted is actually sufficient for $X$ to contain a product subspace:

T19. Let $C(X)$ be an algebra of continuous functions. Then $X$ contains a product subspace if and only if $C(X)$ contains a closed ideal I and two analytic subalgebras $F_{1}$ and $F_{2}$ the closure of whose generated subalgebra is $C(X)$, and whose homomorphic images under the natural homomorphism $C(X) \rightarrow C(X) / I$ are additively related. The product subspace has a nonvacuous interior if and only if the complement of $I$ is non-null.

Proof. The necessity of these conditions appears in the above comments. The sufficiency of the hypotheses will be established if we can show that $C(X) / I$ is equivalent to an algebra of continuous functions defined on a product space, for by $\mathrm{T} 15, C(X) / I$ is equivalent to $C(Y)$ where $I=I(Y)$. We shall show then that the hypotheses of T14 are satisfied for $C(X) / I$.

(a) Since $F_{1}$ and $F_{2}$ are analytic subalgebras of $C(X)$, their homo- 
morphic images $H\left(F_{1}\right)$ and $H\left(F_{2}\right)$ under the natural homomorphism $H: C(X) \rightarrow C(X) / I$ are analytic subalgebras of $C(X) / I$.

(b) By hypothesis the algebras $H\left(F_{1}\right)$ and $H\left(F_{2}\right)$ are additively related.

(c) $\mathcal{C}\left(\left(F_{1} \cup F_{2}\right)_{A}\right)=C(X)$ implies that $\left(F_{1} \cup F_{2}\right)_{A}$ is dense in $C(X)$ which implies that $H\left(\left(F_{1} \cup F_{2}\right)_{A}\right)=\left(H\left(F_{1}\right) \cup H\left(F_{2}\right)\right)_{A}$ is dense in $C(X) / I$ since $H$ is continuous and onto all of $C(X) / I$, and hence $\mathcal{C}\left(\left(H\left(F_{1}\right) \cup H\left(F_{2}\right)\right)_{A}\right)=C(X) / I$.

We have then that the hypotheses of T14 are satisfied for $C(X) / I$, and the first statement of the theorem is therefore proved. The second statement in the theorem follows from T17.

Note that T19 is a generalization of T14 in the sense that if the ideal $I$ in T19 can be taken as the null ideal $(\theta)$, then the hypotheses of T19 are those of T14.

\section{BIBLIOGRAPHY}

1. S. Eilenberg, Banach space methods in topology, Ann. of Math. vol. 43 (1942) pp. 568-579.

2. S. B. Myers, Banach spaces of continuous functions, Ann. of Math. vol. 49 (1948) pp. 132-140.

3. - Spaces of continuous functions, Bull. Amer. Math. Soc. vol. 55 (1949) pp. 402-407.

4. - Normed linear spaces of continuous functions, Bull. Amer. Math. Soc. vol. 56 (1950) pp. 233-241.

5. M. H. Stone, Applications of the theory of Boolean rings to general topology, Trans. Amer. Math. Soc. vol. 41 (1937) pp. 375-481.

UNIVERSITY OF MichigaN 